\title{
Abnormal ultrasound appearance of the amniotic membranes - diagnostic and significance: a pictorial essay
}

\author{
Liana Pleș ${ }^{1,2}$, Romina-Marina Sima ${ }^{1,2}$, Cristina Moisei ${ }^{2}$, Marius Moga $^{3}$, Laura Dracea ${ }^{3}$
}

1"Carol Davila” University of Medicine and Pharmacy, Bucharest, ${ }^{2}$ "Bucur” Maternity, "St. John” Hospital, Bucharest, ${ }^{3}$ Department of Medical and Surgical Specialties, Faculty of Medicine, "Transilvania" University of Brasov, Romania

\section{Abstract}

The obstetrical ultrasound may identify the protrusion of the uterine surface or placental area into the amniotic cavity. The differential diagnosis of this pathology with uterine adhesions, septate uterus, circumvallate placenta, amniotic band or amniotic sheet can be sometimes difficult. The purpose of the pictorial essay is to exemplify the presence of the amniotic sheet and circumvallate placenta in routine obstetrics screening of all trimesters of pregnancy.

Keywords: amniotic sheet; circumvallate placenta; ultrasound
\end{abstract}

\section{Introduction}

Amniotic sheet is a relatively poor recognized condition [1]. The lack in recognition leads to underdiagnosis with no known consequences on pregnancy outcome.

The obstetrical ultrasound may identify the protrusion of the uterine surface or placental area into the amniotic cavity. The differential diagnoses that arise for this condition are: uterine adhesions, septate uterus, circumvallate placenta or amniotic band syndrome [2,3]. Septate uterus is easily recognizable by its fundal position and large insertion [4]. Uterine synechia or adhesions are related to uterine instrumentation or infection [5].

\section{Abnormal ultrasound appearance of the amniotic membranes}

The current literature regarding the outcome of the abnormal appearance of the amniotic membranes is limited to some small case series and case reports due to the low prevalence of this pathology (between $0.14 \%$

Received 21.11.2016 Accepted 30.01.2017

Med Ultrason

2017, Vol. 19, No 2, 211-215

Corresponding author: Marius Alexandru Moga,

56 Nicolae Balcescu street, Brasov, Romania

Phone/Fax : + 0040268412185

E-mail: moga.og@gmail.com and $0.75 \%$ ) [6]. There are few studies that have identified the causes of amniotic sheets and circumvallate placenta $[7,8]$.

\section{Amniotic sheet}

The etiology of the amniotic sheet formation is not fully known, although some authors identified several risk factors among which the uterine synechiae is the most important. The synechiae can be the result of instrumental deliveries, abortions or other uterine maneuvers [4]. A thin membranar septae, attached on the uterine wall by a large triangulate root, formed by both amnion and chorion, coursing towards the opposite uterine wall, and usually with free edge around, is the most frequent ultrasound detected finding [8]. In some cases, the placenta can be inserted or near the sheet. The size and location of the amniotic sheets can be different or multiple (fig 1).

In most of the cases the base of the sheet exhibits vascularization which can be easily identified with Doppler ultrasound. This is an important sign that differentiates the sheet from the amniotic band which is thinner, with no vascularization [9] and is associated with disruptive fetal deformations [10]. Amniotic sheet is considered to be incidental, identified during routine obstetric ultrasound examinations. It is believed to be a type of membrane pathology distinct from the amniotic band because it is not associated with structural defects of the fetus (fig 


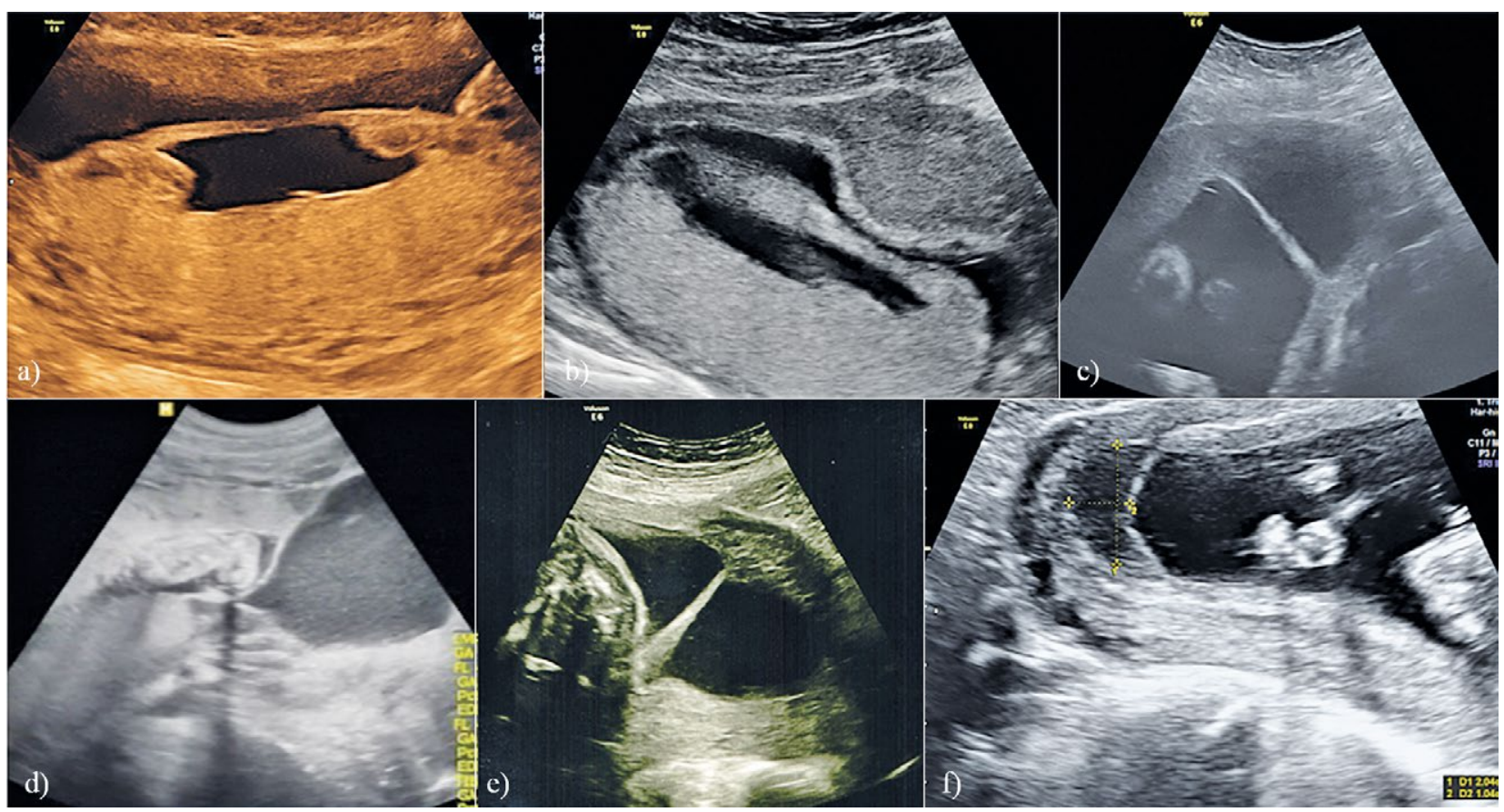

Fig 1. a) Sagittal section of a patient with 16 weeks of pregnancy depicting posterior placenta and an amniotic sheet bonding the two placental edges; b) Similar section of an amniotic sheet at the posterior inserted placenta found at 14 weeks scan. Notice the anterior uterine wall fibroma; c) Fine amniotic sheet at a more advanced pregnancy age placed at the superior placental pole. Note the connexion between the two uterine walls and the less thick appearance due to the amniotic sac expansion; d) Amniotic sheet at the inferior placental pole with the small fetal parts above it. This image can be confused with a placental hematoma but the free movement of the fetal parts around suggests that there is no real separation; e) Typical triangular appearance of the amniotic sheet at 22 weeks with a broad insertion at the placental part; f) Small hematoma at 13 weeks distal to an amniotic sheet (notice the different echogenicity of the collection from the amniotic sac and that the amniotic sheet connects the placental superior pole with the opposite wall).

2) [11]. Torpin et al [12] explained that the rupture of the amnion determine entanglement of various embryonic or fetal parts by fibrous mesodermic bands that results from the chorionic side of the amnion, causing lymphedema, amputation or slash defects, whose location is not genetically determined [12]. Proffitt et al [13] published a case report in which the diagnosis of first-trimester intrauterine fetal demise resulting from decapitation secondary to amniotic band sequence was presented.

\section{Circumvallate placenta}

Another abnormal appearance of the amniotic membranes, but with high impact on the pregnancy outcome is the circumvallate placenta [14]. This pathology represents a morphological abnormality of the placenta in which the chorionic plate is smaller than the basal plate resulting in the folding back of placental and fetal membranes towards the chorionic surface. The prevalence of this abnormality is $0.5 \%$ to $1.8 \%$ [14]. In circumvallate placenta the folded membranes are attached to the placenta and may have a "sheet like" appearance and could be confounded with an amniotic band, clinically being a cause of differential diagnosis of vaginal

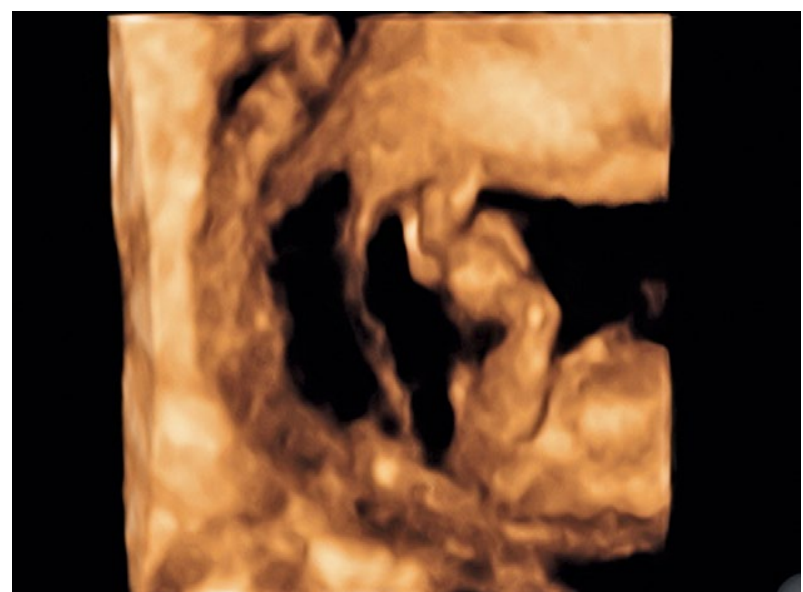

Fig 2. 3D Rendering image at 12 weeks depicting the amniotic sheet at the placental pole

bleeding in the second trimester of pregnancy (fig 3, fig 4) [15].

A particular condition is represented by placental shelves, which are believed to represent circumvallate placentae. The term used for this appearance describes 


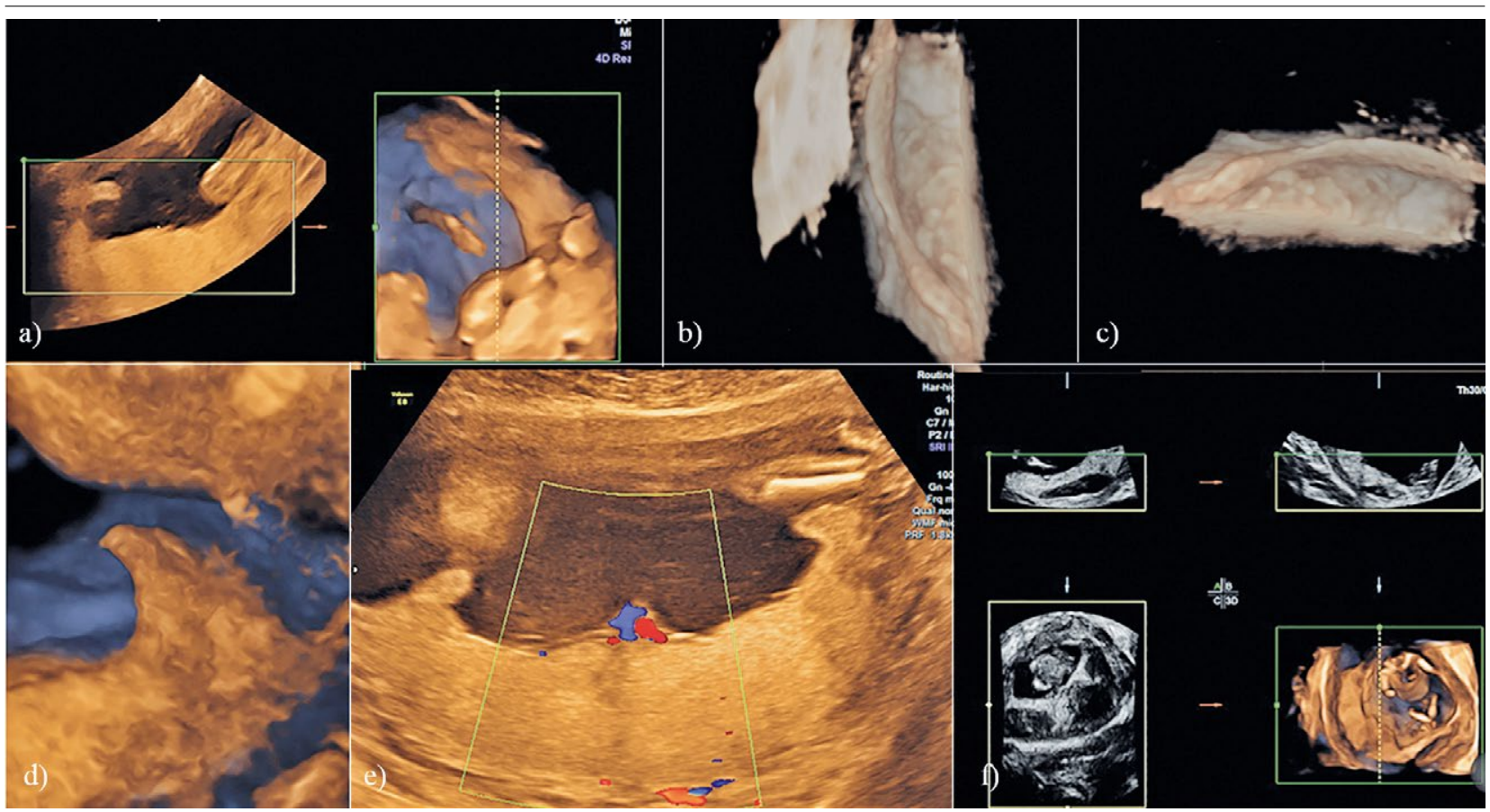

Fig 3. a) 3D rendering image of the circumvallate placenta depicting a small placenta with elevated edges centered by the umbilical cord insertion; b) and c) Rendering 3D images of the amniotic membrane insertion with a ridge like appearance more central than the placental edges; d) 3D rendering image of the inferior circumvallate placental edge. The folded membrane inserted inner than the placental edge; e) sagittal section of the pregnant uterus with posterior placenta circumvallate. The raised placental edges and central umbilical cord insertion can be seen; f) 3D sectional and rendering image of the circumvallate placenta in a 14 weeks pregnancy

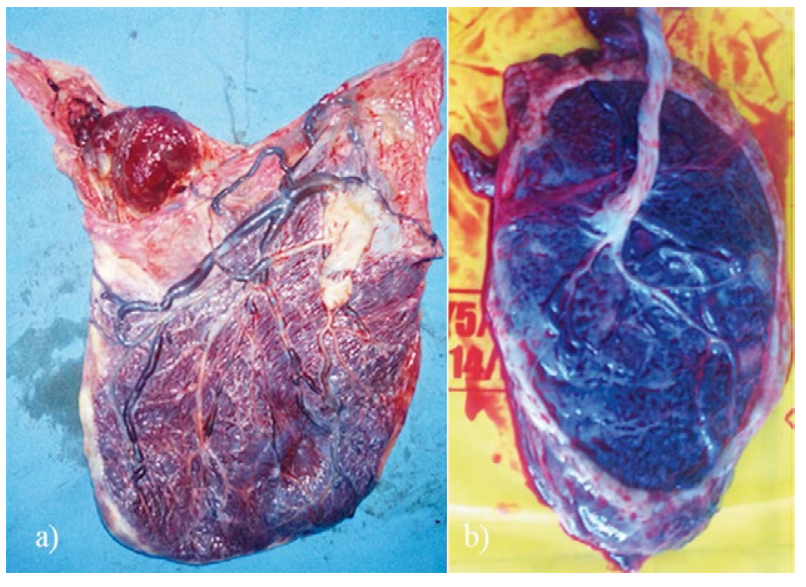

Fig 4. a) Anatomical specimen of the placenta with amniotic sheet (folded superior membrane), aberrant lobe and marginal cord insertion in complicated pregnancy (placental abruption); b) Anatomical specimen of an uncomplicated circumvallate placenta.

a shelf at the margin of the placenta observed in ultrasound. It is a tissue contiguous with the edge of the placenta, protruding into the uterine cavity. It has the same echogenicity with the placenta and has no connections to any fetal parts (fig 5) $[16,17]$.

\section{Ultrasound differential diagnosis}

Circumvallate placental membranes ore original from the fetal edge of the placenta while amniotic sheets attach to the uterine wall itself. There are studies where the utility of Doppler ultrasound for diagnosing the amniotic sheet was demonstrated. In this situation, the arterial flow confirmed the presence of maternal tissue in the sheet [17]. There have been numerous reports on the antenatal diagnosis of circumvallate placenta using two-dimensional (2D) and three-dimensional ultrasound $[16,18,19]$.

Amniotic sheets were associated with malpresentations, higher primary cesarean section rates, and preterm births [6]. However, amniotic sheets represent a benign condition that is not associated with fetal deformity or adverse fetal outcome [20]. Other studies have suggested that amniotic sheets may be associated with a greater likelihood of intrauterine fetal death [10]. In the same time, it was described that third-trimester circumvallate placenta may be associated with preterm delivery, fetal malformations, placental abruption, intrauterine growth restriction and perinatal death [20].

An increased obstetric morbidity was associated with the appearance of amniotic sheets [8]. At the same time, 


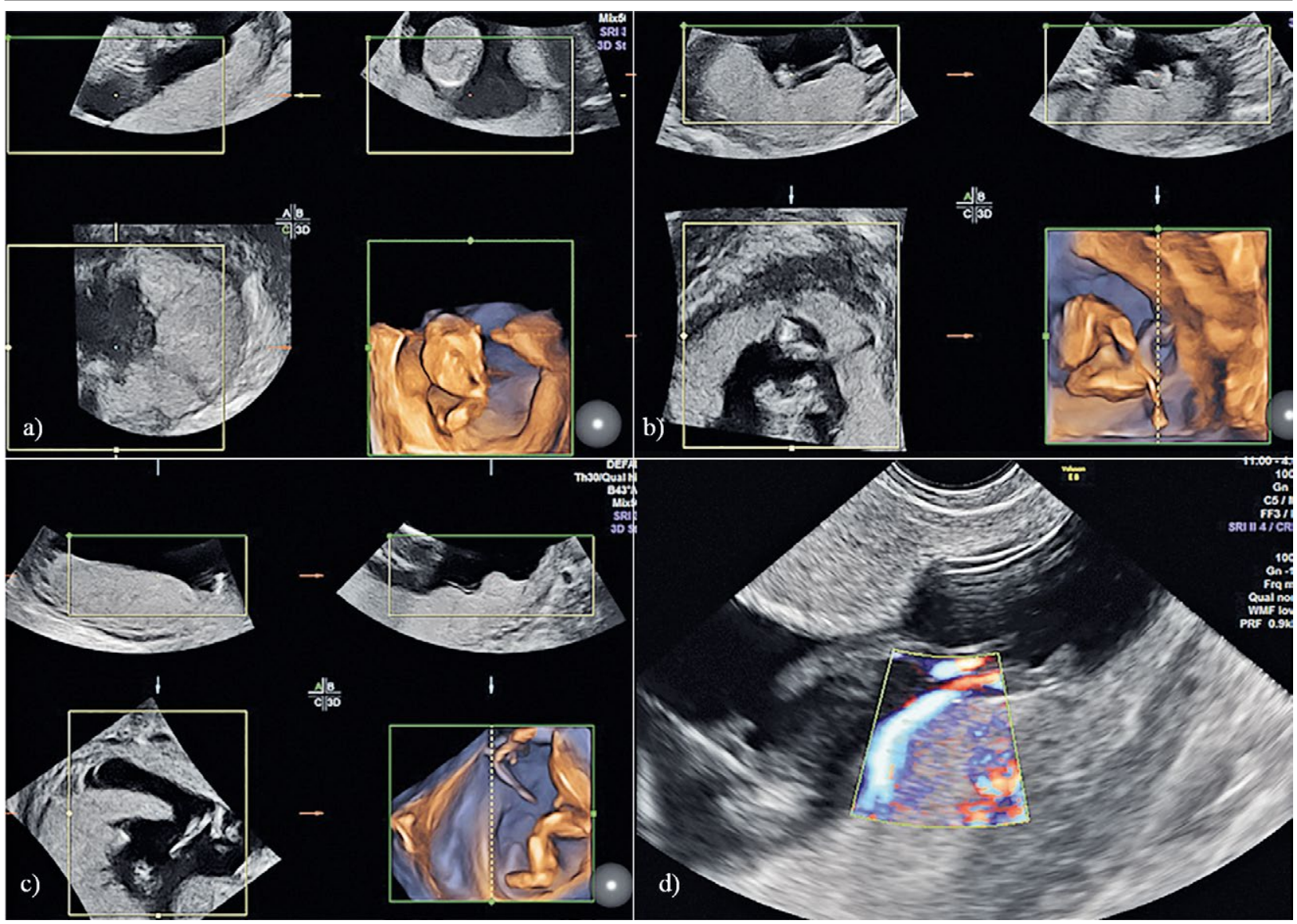

Fig 5. a) $3 \mathrm{D}$ axial planes and rendering of an amniotic shelf at the placental edge, Notice the same echogenicity as the placenta; b) Same patient with a ROI placed in a different section depicting a partial protrusion of the placental ridge with no connection to the fetal parts or the opposite wall; c) 3D axial and rendering image depicting a protruding shelf of the placenta (possible a partial circumvallate placenta; d) Doppler color gate placed at the bottom of the shelf showing vascularization at this level.

it would be premature to change the standard management of these patients, particularly because there are presently no proven strategies to decrease events such as premature rupture of membranes.

Amniotic sheets increase the rates of preterm birth ( $<37$ weeks), breech births, a nuchal cord at delivery, and neonatal intensive care unit admissions. In particular the close monitoring of these patients is needed [21]. It seems that the presence of an amniotic sheet is not associated with an increased risk of amniotic sheets in subsequent pregnancies [21].

\section{Conclusion}

Ultrasound scan in the first two trimesters of pregnancy can reveal some placental and amniotic abnormalities that must be correctly diagnosed in order to offer a proper counseling for the mother. Amniotic sheet and amniotic shelves have been proved to be benign condi- tions with a favorable pregnancy outcome in the majority of the cases. Meanwhile, amniotic band syndrome and circumvallate placenta are important differential diagnosis with potential fetal abnormalities and poor pregnancy outcome.

\section{Conflict of interest: none}

\section{References}

1. Mahony BS, Filly RA, Callen PW, Golbus MS. The amniotic band syndrome: antenatal sonographic diagnosis and potential pitfalls. Am J Obstet Gynecol 1985;152:63-68.

2. Turğal M, Özyüncü Ö, Yazıcıŏlu A, Önderoğlu LS. Integration of three-dimensional ultrasonography in the prenatal diagnosis of amniotic band syndrome: A case report. J Turk Ger Gynecol Assoc 2014;15:56-59.

3. Moerman P, Fryns JP, Vandenberghe K, Lauweryns JM. Constrictive amniotic bands, amniotic adhesions, and limb- 
body wall complex: discrete disruption sequences with pathogenetic overlap. Am J Med Genet 1992;42:470-479.

4. Sato M, Kanenishi K, Ito M, Tanaka H, Takemoto M, Hata T. Antenatal 3-D sonographic features of uterine synechia. J Obstet Gynaecol Res 2013;39:395-398.

5. Ahmadi F, Siahbazi S, Akhbari F, Eslami B,Vosough A. Hysterosalpingography finding in intra uterine adhesion (Asherman's Syndrome): a pictorial essay. Int J Fertil Steril 2013;7:155-160 .

6. Tan KB, Tan TY, Tan JV, Yan YL, Yeo GS. The amniotic sheet: a truly benign condition? Ultrasound Obstet Gynecol 2005;26:639-643 .

7. Tuuli MG, Shanks A, Bernhard L, Odibo AO, Macones GA, Cahill A. Uterine synechiae and pregnancy complications. Obstet Gynecol 2012;119:810-814.

8. Nelson LD, Grobman WA. Obstetric morbidity associated with amniotic sheets. Ultrasound Obstet Gynecol 2010;36:324-327.

9. Ozkavukcu E, Haliloğlu N. Gray-scale and color Doppler US findings of amniotic sheets. Diagn Interv Radiol 2012;18:298-302.

10. Özge K, Canan YT, Mert Y, et al. Amniotic sheet and amniotic band syndrome: pitfalls in distinguishing two cases. Perinat J 2014;22:53-56.

11. Sistrom CL, Ferguson JE. Abnormal membranes in obstetrical ultrasound: incidence and significance of amniotic sheets and circumvallate placenta. Ultrasound Obstet Gynecol 1993;3:249-255.

12. Torpin R, Faulkner A. Intrauterine amputation with the missing member found in the fetal membranes. JAMA 1966;198:185-187.
13. Proffitt E, Phillips M, DeMauro C, Conde K, Powell J. Ultrasonographic diagnosis of intrauterine fetal decapitation secondary to amniotic band sequence: a case report. $\mathrm{J}$ Emerg Med 2016;50:e129-e131.

14. Suzuki S. Clinical significance of pregnancies with circumvallate placenta. J Obstet Gynaecol Res 2008;34:51-54.

15. Arlicot C, Herve P, Simon E, Perrotin F. Three-dimensional surface rendering of the chorionic placental plate: the "tire" sign for the diagnosis of a circumvallate placenta. J Ultrasound Med 2012;31:340-341.

16. Hata T, Fujiwara T, Ishibashi M, Kuwamura E, Tenkumo C, Ishihara G. Antenatal three-dimensional sonographic features of placental shelf. J Med Ultrason 2012;39:4344.

17. AboEllail MA, Kanenishi K, Mori N, Kurobe A, Hata T. HDlive imaging of circumvallate placenta. Ultrasound $\mathrm{Ob}-$ stet Gynecol 2015;46:513-514.

18. Bowman S, Kennedy AM. Sonographic appearance of the placenta. Curr Prob Diagn Radiol 2014;43:356-373.

19. Shen O, Golomb E, Lavie O, Goldberg Y, Eitan R, Rabinowitz RR. Placental shelf - a common, typically transient and benign finding on early second-trimester sonography. Ultrasound Obstet Gynecol 2007;29:192-194.

20. Anyikam AL, Hull AD, Benke S, Trivedi N, LaCoursiere DY, Pretorius DH. Prenatal diagnosis of circumvallate placenta and pregnancy outcomes. Obstet Gynecol 2014;123 Suppl 1:98S.

21. Gün İ, Muhçu M, Müngen E, Kiliç S, Atay V. Effect of an amniotic sheet on pregnancy outcomes. J Ultrasound Med 2013;32:807-813. 